\title{
Ultrasound Therapy
}

National Cancer Institute

\section{Source}

National Cancer Institute. Ultrasound Therapy. NCI Thesaurus. Code C28003.

A form of treatment that uses very high frequency sound waves for the purpose of treating an injury or illness. 